\title{
Treating the elderly patient with chronic lymphocytic leukemia: current and emerging options
}

This article was published in the following Dove Press journal:

Blood and Lymphatic Cancer:Targets and Therapy

19 May 2014

Number of times this article has been viewed

\section{Zahra Mozaheb}

Department of HematologyOncology, Imam-Reza Hospital, Mashhad University of Medical Science, Mashhad, Iran
Correspondence: Zahra Mozaheb Department of Hematology-Oncology, Imam-Reza Hospital, Mashhad University of Medical Science, Mashhad, Iran Email mozahebz@mums.ac.ir
Abstract: Management of cancer in the elderly is an increasingly common problem. The risk of chronic lymphocytic leukemia (CLL) increases significantly after the age of 65 years. One of the most important prognostic factors in the elderly is the burden of comorbidity. Severe and multiple comorbidities significantly decrease survival in patients with CLL. Therefore, not only age but also the incidence and severity of comorbidity should influence the treatment strategy for patients at an individual level. This review attempts to identify the best management strategies for elderly patients with CLL based on individual functional reserve and estimation of individual life expectancy.

Keywords: chronic lymphocytic leukemia, elderly, management

\section{Introduction}

Management of cancer in the elderly is a common problem. Aging may be interpreted as a progressive loss of stress tolerance due to a decline in the functional reserve of multiple organ systems, a high prevalence of comorbid conditions, reduced cognition, limited socioeconomic support, and a high prevalence of depression. Aging is a highly individualized process, and chronologic age may not reflect life expectancy or functional reserve at the individual level. A comprehensive geriatric assessment is available to accommodate the diversity of the geriatric population. ${ }^{1}$

Chronic lymphocytic leukemia (CLL) is a disease of mature B lymphocytes, and is more common in the elderly. ${ }^{2}$ It is markedly more common in patients over the age of 65 years, with an incidence of 22-30 per 100,000 in Western countries. ${ }^{3}$ According to the Surveillance Epidemiology and End Results registry, 75\% of patients are older than 65 years at the time of diagnosis. Not all CLL patients are eligible for aggressive upfront chemoimmunotherapy regimens, so what is the optimal treatment approach for the more frail elderly patient?

Despite the life expectancy of at least 10 years in patients with early-stage CLL, the disease remains an incurable one, especially in elderly patients, and evidence-based therapeutic strategies are still lacking for this age group and need more investigation. Survival is significantly impaired in elderly patients with CLL because of age and multiple comorbidities. ${ }^{3}$ In one study, $89 \%$ of patients with CLL had one or more comorbidities at diagnosis and $46 \%$ had at least one major comorbidity. ${ }^{4}$ The best initial treatment for elderly patients with CLL has not yet been defined. Treatment decisions in this age group should be taken carefully, considering not only the stage of and risk factors for CLL but also the age and performance status of the patient, ${ }^{5}$ comorbid 
conditions, social environment, and any contraindications to therapy. ${ }^{3,6}$ Although patients used to be classified based on age alone, it is now well recognized that chronologic age is not a reliable proxy for physiologic age or fitness. ${ }^{7}$ Comprehensive geriatric assessment of functional status is one strategy used to evaluate fitness/frailty more accurately. Standardized scales are available to perform a comprehensive geriatric assessment, and these techniques have been shown to reliably predict the ability of elderly patients to tolerate chemotherapy. ${ }^{8}$ The pharmacokinetics (absorption, metabolism, excretion) of the drugs to be used should also be considered when evaluating the suitability of a particular treatment approach. ${ }^{9}$ The presence of two or more comorbid conditions was associated with shorter progression-free and overall survival in the German CLL5 trial. ${ }^{10}$ Therefore, we should use these tools to distinguish between physically fit and unfit patients when managing CLL in the elderly.

\section{Management of early-stage disease}

The standard approach to treatment of patients with early disease (Binet stage A and B without active disease; Rai 0, I, and II without symptoms) is a watch-and-wait strategy. Complete blood cell counts and clinical examinations should be done every 3 to 12 months. ${ }^{11}$ The results of a previous randomized trial indicate that chlorambucil can delay disease progression, even though it does not affect survival; the differences in survival 9 years after randomization between untreated patients with progression to stage $\mathrm{B}$ and treated patients with progression to stage $\mathrm{B}$ ( $54 \%$ versus $21 \%$, respectively) suggest that early exposure to the drug selects for resistant clones, which are the cause of the poor prognosis in patients who have no response to early therapy. ${ }^{12}$

A meta-analysis showed that treating CLL patients with indolent disease does not prolong survival. However, patients with p53 abnormalities at diagnosis have an extremely poor prognosis, and treatment with an alemtuzumab-containing regimen as first-line therapy, regardless of stage, is becoming acceptable. ${ }^{6}$ In elderly patients, intravenous alemtuzumab is associated with adverse reactions and infectious morbidity, but subcutaneous low-dose alemtuzumab seems to minimize these adverse effects. ${ }^{10}$ The CLL 7 protocol used by the French and German study group suggests that patients with early-stage disease and more than one of four unfavorable risk factors (unfavorable cytogenetics, elevated serum thymidine kinase, nonmutated immunoglobulin heavy chain variable status, and short lymphocyte doubling time) may benefit from early treatment. ${ }^{5,13}$
The molecular profile of CLL predicts time to progression, time to need for therapy, and overall survival. Decision-making in clinical practice should be based on symptoms and clinical features of the disease, and the use of molecular profiling in management of CLL remains a research question. The only clear exception to this is in symptomatic patients with del(17p) or p53 mutations, which may dictate therapy. These patients should be treated with agents that act independently of p53. ${ }^{14}$ The more cost-effective and established markers of the disease, such as $\beta-2$ microglobulin, are used by some clinicians, and can be incorporated into nomograms for assessment of the risk of progression. ${ }^{5}$

\section{Management of advanced disease}

The indications for initiating treatment in older patients with CLL are the same as those for younger patients. ${ }^{9,15}$ Staging of the disease is assessed according to the Rai and Binet classifications and the need for treatment according to National Cancer Institute criteria. These include the patients where in Binet stage $\mathrm{C}$ and in stage $\mathrm{B}$ or $\mathrm{A}$ if they had rapid disease progression (lymphocyte doubling time less than 3 months) or symptoms from enlarged lymph nodes and organs, and/or if they had severe B symptoms. ${ }^{10}$

Before initiating treatment in elderly patients, we need to consider a number of issues. First, recurrent infections are not a reason to initiate therapy, because treatment increases rather than decreases the risk of infection; instead, these patients should undergo assessment for hypogammaglobulinemia or other causes of immunocompromise. ${ }^{15}$ Second, elderly patients should receive aggressive supportive care measures, including age-appropriate cancer screening, to decrease the risk of disease-related complications. ${ }^{16}$ Third, several studies have shown that low vitamin D levels may be associated with more rapid CLL progression and shorter survival time, but it is unknown if vitamin D replacement reverses this problem. ${ }^{17}$ In patients at risk, assessment of vitamin $\mathrm{D}$ at the time of diagnosis can be considered part of good general care for elderly patients. ${ }^{18}$ Fourth, vaccination strategies should be used to reduce the risk of infection in CLL patients. It is suggested that all immunocompromised patients, including those with leukemia, receive initial pneumococcal vaccination and annual influenza vaccination, although live attenuated vaccines are contraindicated in patients with CLL. ${ }^{19}$ Fifth, the presence of comorbid health conditions and the ability of elderly patients to tolerate chemotherapy should be considered. Finally, after adjusting for disease stage, the fitness and comorbidity of the patient should be assessed when determining the best treatment. 
The Mayo Clinic cohort study showed that immunoglobulin heavy chain variable mutation status, ZAP-70, and CD38 were powerful predictors in patients of all ages, including those aged older than 75 years. ${ }^{20}$

Catovsky et al suggested that fludarabine + cyclophosphamide should be the standard treatment for CLL in all groups and the basis for new protocols that incorporate monoclonal antibodies. ${ }^{21}$ Improved survival has recently been demonstrated following first-line immunochemotherapy with FCR (fludarabine + cyclophosphamide + rituximab) in physically fit patients with CLL; therefore, in patients who are physically active, with no major health problems and normal renal function, FCR is now the standard first-line therapy. In patients with relevant comorbidity, chlorambucil remains the standard therapy. Alternatives include dose-reduced purine analogbased therapies (fludarabine + cyclophosphamide, FCR) or bendamustine. ${ }^{11}$ Although fludarabine produces the highest remission rates in patients with B-cell CLL, responses are often complicated by an increased risk of infection due to neutropenia, decreased CD4 lymphocytes, and the immunosuppressive effect of corticosteroids, particularly in elderly patients. Marotta et al have suggested that corticosteroids should be avoided and the doses of both fludarabine and cyclophosphamide should be reduced in order to minimize the risk of infectious complications. ${ }^{22}$ Because maintenance of good quality of life is the main goal of treatment in elderly patients with CLL, dose reduction of fludarabine, appropriate use of myeloid growth factors, and prophylactic antibiotics appear to be mandatory. ${ }^{23}$

A study by Eichhorst et al suggests that first-line therapy with fludarabine is not superior to chlorambucil with regard to progression-free survival and overall survival, although it does achieve higher response rates and delays time to treatment failure in elderly patients with CLL. ${ }^{10}$ Woyach et al also recommend chlorambucil as an acceptable treatment for older patients with CLL and suggest that rituximab is beneficial regardless of age. ${ }^{24}$

When used as a first-line treatment for patients with CLL, alemtuzumab achieved significantly improved progressionfree survival, a longer time until the need for alternative treatment, increased overall and complete response rates, and minimal residual disease-negative remissions compared with chlorambucil, with predictable and manageable toxicity. ${ }^{25}$

Kay et al demonstrated that a novel regimen of pentostatin, cyclophosphamide, and rituximab in previously untreated CLL patients had significant clinical activity despite poor risk-based prognoses, achieved minimal residual disease, and had modest toxicity. This regimen was equally effective in younger and older ( $>70$ years) patients and also in del(11q22.3) versus other favorable prognostic factors. ${ }^{26}$

In a study evaluating the long-term survival of elderly CLL patients treated initially with lenalidomide, Strati et al reported responses lasting more than 3 years in 58\% of patients, and overall survival in this study compared favorably with the historical experience. Patients with long-term responses to lenalidomide had lower baseline plasma $\beta$-2-microglobulin levels, were more likely to have trisomy 12 , and were less likely to have the $17 \mathrm{p}$ deletion. However, a possible correlation between lenalidomide and development of second cancer may limit widespread use of lenalidomide in the treatment of CLL. ${ }^{27}$

The CLL5 trial and the study by Woyach et $\mathrm{al}^{24}$ demonstrated that chlorambucil is a reasonable chemotherapeutic backbone on which to design new combination regimens in elderly patients. The combination of chlorambucil and rituximab is certainly a standard therapeutic option for older patients, ${ }^{28}$ and may be an ideal platform on which to add new targeted agents to improve efficacy. ${ }^{22}$

The US Food and Drug Administration has approved obinutuzumab (GazyvaTM; Genentech Inc., South San Francisco, CA, USA) for use in combination with chlorambucil to treat patients with previously untreated CLL. ${ }^{29}$ The CLL11 trial reported that a combination of obinutuzumab and chlorambucil achieved a statistically significant and clinically meaningful prolongation of progression-free survival, a higher complete response rate, and a minimal residual disease negativity rate compared with a combination of rituximab and chlorambucil, and also showed that the obinutuzumab-chlorambucil combination prolonged overall survival compared with chlorambucil alone. Gazyva achieved an $84 \%$ reduction in the risk of CLL progression, relapse, or death when combined with chlorambucil versus chlorambucil alone (hazard ratio 0.16); the overall response rate was $75.9 \%$ for the Gazyva-treated arm and $32.1 \%$ for the chlorambucil only arm, and the complete response rate was $27.8 \%$ and $0.9 \%$, respectively. ${ }^{30}$

In a study by Fischer et al in CLL patients aged 70 years or older who received bendamustine-rituximab chemoimmunotherapy as first-line treatment of CLL, the overall response rate was $88.0 \%$, with a complete response rate of $23.1 \%$ and a partial response rate of $64.9 \%$. These authors suggested that bendamustine-rituximab chemoimmunotherapy is safe and effective in patients with previously untreated CLL. ${ }^{31}$

These data, along with the knowledge that a majority of CLL patients have comorbid conditions and more than half have at least one major comorbidity, underscore the fact that 
selection of therapy must be individualized. The efficacy and toxicity of the treatment regimen, the mechanism of drug elimination, and the patient's underlying organ function are important considerations. ${ }^{9}$ FCR is now the standard first-line therapy in elderly patients who are physically active with no major health problems and normal renal function. In older patients with major comorbidity, chlorambucil remains the standard therapy. Alternatives are bendamustine or dosereduced purine analog-based therapies. ${ }^{32}$ The appropriate approach suggested for management of previously untreated CLL patients is shown in Figure $1 .^{14}$

In second-line therapy for refractory or relapsed CLL patients, if the relapse or progression occurs at least 12 months after the initial therapy and 24 months after immunochemotherapy, the first-line treatment may be repeated, and if the relapse occurs within 12 months after monotherapy or 24 months after immunochemotherapy, or if the disease does not respond to first-line monotherapy, the therapeutic regimen should be changed. ${ }^{32}$ Chlorambucil-treated patients may be treated with fludarabine as second-line therapy. ${ }^{10}$ Other suggestions include

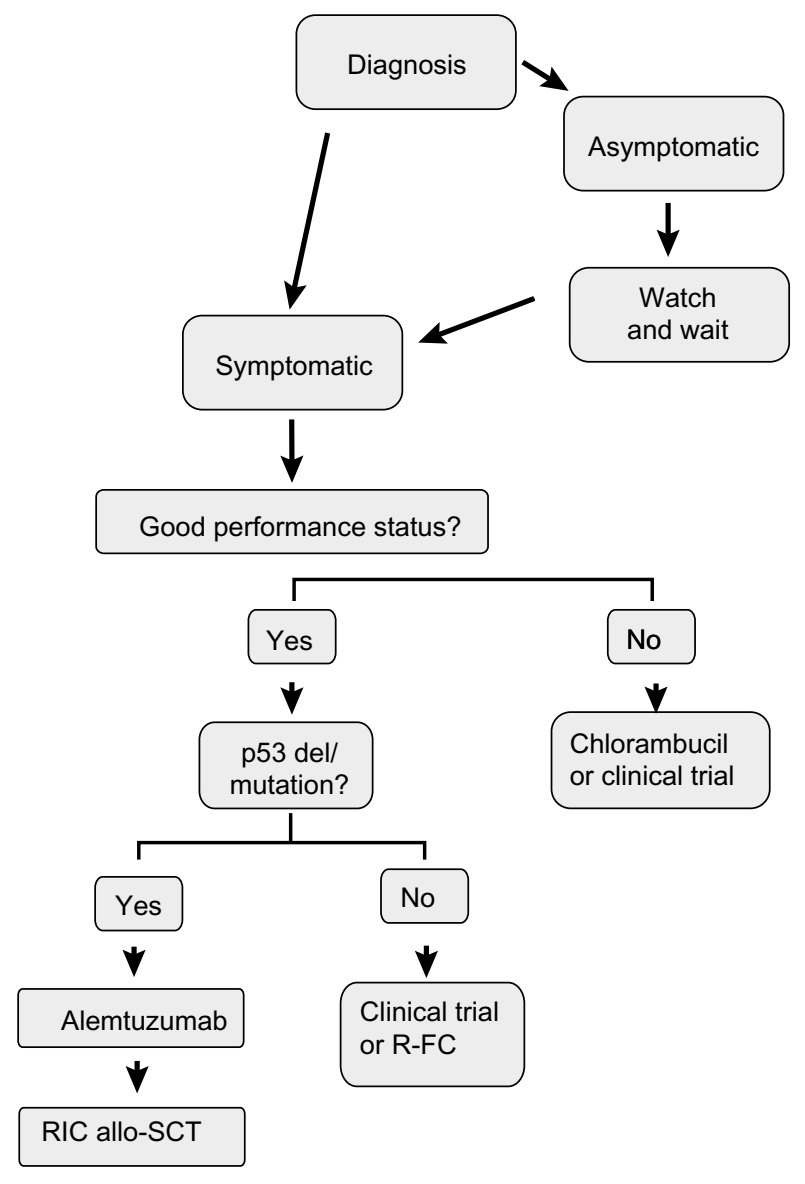

Figure I Appropriate approach for untreated chronic lymphocytic leukemia. Abbreviations: RIC, reduced-intensity conditioning; allo-SCT, allogeneic stem cell transplantation; R-FC, ritoximab-fludarabin, cyclophosphamid. alemtuzumab in physically unfit patients with del(17p) and an alemtuzumab-containing regimen followed by allogeneic stem cell transplantation in physically fit patients. FCR is an appropriate option for patients who are refractory to or have relapsed on first-line therapy with an alkylating agent. Bendamustine or an alemtuzumab-containing regimen is recommended in physically unfit patients without $\operatorname{del}(17 \mathrm{p}) .{ }^{32}$

\section{Stem cell transplantation in elderly CLL patients}

Patients with the del(17p) chromosomal defect frequently do not respond to conventional chemotherapy with chlorambucil or fludarabine + cyclophosphamide, and progression-free survival of these patients remains short, even after FCR therapy. Therefore, physically fit patients should be offered allogeneic stem cell transplantation within the setting of clinical trials, ${ }^{32}$ but this might not be a good option in people who are older or have other health problems because of life-threatening complications and side effects. Older patients may be able to undergo a nonmyeloablative (mini) transplant, (where they receive lower doses of chemotherapy and radiation that do not completely destroy the bone marrow cells), but receive a higher dose of immunosuppressive therapy. ${ }^{33}$ Autologous stem cell transplantation is being investigated in patients with CLL, but it is not clear if this improves survival when compared with standard treatment. The use of stem cell transplantation should be weighed against the risks of the underlying disease, particularly in the setting where improvements in treatment lead to an improved outcome. ${ }^{34}$

\section{Novel agents}

Ibrutinib is an orally administered covalent inhibitor of Bruton tyrosine kinase. A study by O'Brien et $\mathrm{al}^{35}$ has assessed the safety and activity of ibrutinib in treatment-naïve patients aged 65 years and older with CLL. Toxicity was mainly mild to moderate, with one patient developing grade 3 neutropenia and another developing grade 4 thrombocytopenia. After a median follow-up of 22.1 months, 22 (71\%) of 31 patients had achieved an objective response (95\% confidence interval 52.0-85.8); four patients (13\%) had a complete response, and 17 (55\%) patients had a partial response. The authors of this study considered that the safety and activity of ibrutinib in elderly, previously untreated, symptomatic CLL patients was encouraging and merits further investigation in Phase III trials.

Phosphatidylinositol 3-kinase-delta $(\mathrm{PI} 3 \mathrm{~K} \delta)$ is critical for proliferation, activation, and survival of B cells and has a role in homing in lymphoid tissues. Idelalisib is a selective oral inhibitor of $\mathrm{PI} 3 \mathrm{~K} \delta$, and when used in combination with rituximab in 19 relapsed/refractory patients with CLL, the 
overall response rate was $78 \%$. The other study showed that a combination of idelalisib and rituximab is highly active, resulting in durable disease control in older treatment-naïve patients with CLL. These results support further development of idelalisib as front-line treatment for CLL. ${ }^{36}$

Ofatumumab is a monoclonal antibody that targets an epitope encompassing the membrane-proximal small-loop on the CD20 molecule, which differs from the binding site of rituximab. Studies of ofatumumab have shown that it is significantly more effective than rituximab at corresponding dose levels in B cell lines, especially those with low copy numbers of CD20. In CLL patients refractory to fludarabine with bulky lymphadenopathy; refractory to both fludarabine and alemtuzumab and, therefore less suitable for treatment with the CD52 monoclonal antibody alemtuzumab; results from the planned interim analysis showed an encouraging response rate with ofatumumab. ${ }^{37}$

\section{Conclusion}

The treatment decision in elderly patients with CLL is more complex than that in younger patients because of the greater frequency of comorbidities and functional impairment. Therefore, management of CLL in elderly patients is chosen for each patient individually, considering not only the staging and risk factors for the disease but also the patients' physical condition and their drug tolerability. They may not be able to tolerate regimens of the same intensity as well as younger patients, but fit elderly adults should be treated with the goal of achieving a reasonable depth of remission and a progression-free survival of several years. Several rituximabcontaining regimens are appropriate treatment options. Clinical trials specifically designed for elderly patients are urgently needed, and enrolment of patients in dedicated trials is recommended. New nonchemotherapeutic drugs should also be kept in mind in this setting.

\section{Disclosure}

The author reports no conflict of interest in this work.

\section{References}

1. Balducci L, Extermann M. Management of cancer in the older person: a practical approach. Oncologist. 2000;5(3):224-237.

2. Mozaheb Z, Aledavood A, Farzad F. Distributions of major sub-types of lymphoid malignancies among adults in Mashhad, Iran. Cancer Epidemiol. 2011;35(1):26-29.

3. Eichhorst B, Goede V, Hallek M. Treatment of elderly patients with chronic lymphocytic leukemia. Leuk Lymphoma. 2009;50(2):171-178.

4. Thurmes P, Call T, Slager S, et al. Comorbid conditions and survival in unselected, newly diagnosed patients with chronic lymphocytic leukemia. Leuk Lymphoma. 2008;49(1):49-56.

5. Mozaheb Z, NazarAbadi MH,Afzal M. Chronic lymphocytic leukemia and prognostic factors. Asian Pac J Cancer Prev. 2012;13(7):3009-3013.
6. Auer RL, Gribben J, Cotter FE. Emerging therapy for chronic lymphocytic leukaemia. Br J Haematol. 2007;139(5):635-644.

7. Tsimberidou AM, Wen S, O'Brien S, et al. Assessment of chronic lymphocytic leukemia and small lymphocytic lymphoma by absolute lymphocyte counts in 2,126 patients: 20 years of experience at the University of Texas MD Anderson Cancer Center. J Clin Oncol. 2007;25(29):4648-4656.

8. Wierda WG, O'Brien S, Wang X, et al. Prognostic nomogram and index for overall survival in previously untreated patients with chronic lymphocytic leukemia. Blood. 2007;109(11):4679-4685.

9. Shanafelt T. Treatment of older patients with chronic lymphocytic leukemia: key questions and current answers. Hematology Am Soc Hematol Educ Program. 2013;2013(1):158-167.

10. Eichhorst BF, Busch R, Stilgenbauer S, et al. First-line therapy with fludarabine compared with chlorambucil does not result in a major benefit for elderly patients with advanced chronic lymphocytic leukemia. Blood. 2009;114(16):3382-3391.

11. Eichhorst B, Dreyling M, Robak T, Montserrat E, Hallek M. Chronic lymphocytic leukemia: ESMO clinical practice guidelines for diagnosis, treatment and follow-up. Ann Oncol. 2011;22(6):vi50.

12. Dighiero G, Maloum K, Desablens B, et al. Chlorambucil in indolent chronic lymphocytic leukemia. N Engl J Med. 1998;338(21): 1506-1514.

13. Montillo M, Hamblin T, Hallek M, Montserrat E, Morra E. Chronic lymphocytic leukemia: novel prognostic factors and their relevance for risk-adapted therapeutic strategies. Haematologica. 2005;90(3): 391-399.

14. Gribben JG. How I treat CLL up front. Blood. 2010;115(2):187-197.

15. Hallek M, Cheson BD, Catovsky D, et al. Guidelines for the diagnosis and treatment of chronic lymphocytic leukemia: a report from the International Workshop on Chronic Lymphocytic Leukemia updating the National Cancer Institute Working Group 1996 guidelines. Blood. 2008;111(12):5446-5456.

16. Shanafelt TD, Kay NE. Comprehensive management of the CLL patient: a holistic approach. Hematology Am Soc Hematol Educ Program. 2007:324-331.

17. Shanafelt TD, Drake MT, Maurer MJ, et al. Vitamin D insufficiency and prognosis in chronic lymphocytic leukemia. Blood. 2011;117(5): 1492-1498.

18. Kennel KA, Drake MT, Hurley DL. Vitamin D deficiency in adults: when to test and how to treat. Mayo Clin Proc. 2010;85(8):752-757.

19. Swartz MH. Textbook of Physical Diagnosis: History and Examination With DVD. 6th ed. Philidelphia, PA, USA: Elsevier Health Sciences; 2014.

20. Shanafelt TD, Rabe KG, Kay NE, et al. Age at diagnosis and the utility of prognostic testing in patients with chronic lymphocytic leukemia. Cancer. 2010;116(20):4777-4787.

21. Catovsky D, Richards S, Matutes E, et al. Assessment of fludarabine plus cyclophosphamide for patients with chronic lymphocytic leukaemia (the LRF CLL4 Trial): a randomised controlled trial. Lancet. 2007;370(9583):230-239.

22. Marotta G, Bigazzi C, Lenoci M, Tozzi M, Bocchia M, Lauria F. Low-dose fludarabine and cyclophosphamide in elderly patients with B-cell chronic lymphocytic leukemia refractory to conventional therapy. Haematologica. 2000;85(12):1268-1270.

23. Shvidel L, Shtalrid M, Bairey O, et al. Conventional dose fludarabine-based regimens are effective but have excessive toxicity in elderly patients with refractory chronic lymphocytic leukemia. Leuk Lymphoma. 2003;44(11):1947-1950.

24. Woyach JA, Ruppert AS, Rai K, et al. Impact of age on outcomes after initial therapy with chemotherapy and different chemoimmunotherapy regimens in patients with chronic lymphocytic leukemia: results of sequential cancer and leukemia group B studies. J Clin Oncol. 2013;31(4):440-447.

25. Hillmen P, Skotnicki AB, Robak T, et al. Alemtuzumab compared with chlorambucil as first-line therapy for chronic lymphocytic leukemia. J Clin Oncol. 2007;25(35):5616-5623. 
26. Kay NE, Geyer SM, Call TG, et al. Combination chemoimmunotherapy with pentostatin, cyclophosphamide, and rituximab shows significant clinical activity with low accompanying toxicity in previously untreated B chronic lymphocytic leukemia. Blood. 2007;109(2):405-411.

27. Strati P, Keating MJ, Wierda WG, et al. Lenalidomide induces long-lasting responses in elderly patients with chronic lymphocytic leukemia. Blood. 2013;122(5):734-737.

28. Hillmen P, Gribben JG, Follows GA, et al. Rituximab plus chlorambucil in patients with CD20-positive B-cell chronic lymphocytic leukemia (CLL): final response analysis of an open-label phase II study. Lancet. 2007;370(9583):230-239.

29. HiGiulio S. FDA approves Gazyva for chronic lymphocytic leukemia. Oncology Times. 2013. Available from: http://www.fda.gov/ newsevents/newsroom/pressannouncements/ucm373209.htm. Accessed April 4, 2014.

30. Stockwell S. ONLINE FIRST: CLL11 trial called game changer for treating elderly patients. Oncology Times. 2014. Available from: http://journals.lww.com/oncology-times/blog/onlinefirst/pages/post. aspx?PostID=947. Accessed April 4, 2014.

31. Fischer K, Cramer P, Busch R, et al. Bendamustine in combination with rituximab for previously untreated patients with chronic lymphocytic leukemia: a multicenter phase II trial of the German Chronic Lymphocytic Leukemia Study Group. J Clin Oncol. 2012;30(26):3209-3216.
32. Eichhorst B, Hallek M, Dreyling M. Clinical practice guidelines. Ann Oncol. 2010;21(5):v162-v164.

33. Dreger P, Corradini P, Kimby E, et al. Indications for allogeneic stem cell transplantation in chronic lymphocytic leukemia: the EBMT transplant consensus. Leukemia. 2007;21(1):12-17.

34. Gribben JG. Stem cell transplantation in chronic lymphocytic leukemia. Biol Blood Marrow Transplant. 2009;15(1):53-58.

35. O'Brien S, Furman RR, Coutre SE, et al. Ibrutinib as initial therapy for elderly patients with chronic lymphocytic leukaemia or small lymphocytic lymphoma: an open-label, multicentre, phase $1 \mathrm{~b} / 2$ trial. Lancet Oncol. 2014;15(1):48-58.

36. O'Brien SM, Lamanna N, Kipps TJ, et al. A phase II study of the selective phosphatidylinositol 3-kinase delta (PI3K $)$ ) inhibitor idelalisib (GS-1101) in combination with rituximab (R) in treatment-naive patients (pts) $\geq 65$ years with chronic lymphocytic leukemia (CLL) or small lymphocytic lymphoma (SLL). J Clin Oncol. 2013;31 Suppl: Abstr 7005.

37. O'Brien S, Österborg A. Ofatumumab: a new CD20 monoclonal antibody therapy for B-cell chronic lymphocytic leukemia. Clin Lymphoma Myeloma Leuk. 2010;10(5):361-368.
Blood and Lymphatic Cancer: Targets and Therapy

\section{Publish your work in this journal}

Blood and Lymphatic Cancer: Targets and Therapy is an international, peer-reviewed, open access journal focusing on blood and lymphatic cancer research, identification of therapeutic targets and the optimal use of preventative and integrated treatment interventions to achieve improved outcomes, enhanced survival and quality of life for the

\section{Dovepress}

cancer patient. The manuscript management system is completely online and includes a very quick and fair peer-review system. Visit http://www.dovepress.com/testimonials.php to read real quotes from published authors. 